\title{
Evaluación y acreditación de titulaciones universitarias en Educación desde el punto de vista del profesorado
}

\author{
Francisco Javier IBÁÑEZ-LÓPEZ \\ Fuensanta HERNÁNDEZ-PINA \\ Fuensanta MONROY
}

\begin{abstract}
Datos de contacto:
Francisco Javier Ibáñez-López Universidad de Murcia. fjil@um.es

Fuensanta Hernández-Pina Universidad de Murcia fhpina@um.es

Fuensanta Monroy Universidad de Murcia fuensanta.monroy@um.es
\end{abstract}

Recibido: 20/02/2020 Aceptado: 25/07/2020

\begin{abstract}
RESUMEN
Las titulaciones de Educación Superior deben ser sometidas a procesos de evaluación de la acreditación para asegurar su correcto funcionamiento y cumplimiento de objetivos. Esta investigación tuvo por objetivo analizar las facilidades y dificultades encontradas a la hora de realizar los procesos de evaluación y acreditación por parte del profesorado y comprobar el liderazgo que los equipos de dirección de los centros y los coordinadores de titulaciones ejercen sobre los mismos. Fue desarrollada en un centro universitario estatal que consiguió finalizar en 2018 la acreditación por parte de la Agencia Nacional de Evaluación de la Calidad y Acreditación (ANECA) de todas sus titulaciones oficiales. El estudio fue descriptivo y se abordó con un enfoque de investigación mixto mediante la recogida de información cuantitativa y cualitativa, a través de una encuesta tipo escala Likert y entrevistas semiestructuradas. Los resultados señalaron tres dificultades principales: la complejidad de los procedimientos en sí, la falta de personal especializado para llevar a cabo estos procedimientos y en general, la poca participación de los grupos de interés. Se destacaron las facilidades que ofrece el servicio de gestión de la Calidad de la universidad, y el liderazgo que abanderan el equipo directivo y los coordinadores de las titulaciones. Las conclusiones certificaron una necesidad de simplificar y modificar los procesos, de apostar por su eficiencia con más medios materiales y humanos, y de fomentar la participación activa de todos los grupos de interés.
\end{abstract}

PALABRAS CLAVE: Acreditación (educación); evaluación; Educación Superior; Calidad de la educación. 


\title{
Teachers' viewpoint about evaluation and accreditation of Education degrees
}

\begin{abstract}
University degrees must be evaluated in order to ensure they function properly and reach their objectives. This study aimed at finding out about lecturers' opinion about various aspects of the evaluation process implemented at their university, and about the leadership of the management and coordination teams. The focus of study was the faculty of education of a state university whose degrees had been positively evaluated by National Agency for Quality Assessment and Accreditation (ANECA) in 2018. A mixed method approach was used as opinions were collected from a large number of teachers via a Likert scale, as well as from a small sample of teachers in face-to-face interviews. The results revealed that lecturers identified three main difficulties in evaluation: procedures are complex, a lack of specialized staff to evaluate, and low participation of all stakeholders. Participants enhanced the role played by the university Quality Service and the leadership of the management and coordination teams of the faculty. The results showed there is a need for simpler evaluation, additional material and human resources, and greater involvement and active participation of all stakeholders.
\end{abstract}

KEYWORDS: Accreditation (education); evaluation; Higher Education; Quality of education.

\section{Introducción}

Los procesos de evaluación y acreditación de las titulaciones de Educación Superior son un complejo e intenso procedimiento que conlleva la recogida de información (evidencias) y su procesado e interpretación para la elaboración de memorias, autoinformes y preparación de visitas de paneles externos. Estos son llevados a cabo por todo el personal relacionado con las titulaciones, alumnado, profesorado, egresados, empleadores y personal de administración y servicios (grupos de interés). Una vez finalizados, se debe aplicar las modificaciones y propuestas de mejora necesarias con el objetivo de mejorar la calidad de los estudios y las prestaciones que son propias de las instituciones universitarias, lo cual permite perseguir alcanzar la excelencia (Rodríguez Espinar, 2013).

Estos procesos tienen su origen en la década de los 90 del siglo pasado con el desarrollo de programas piloto para evaluar la Calidad de la Enseñanza Superior. Fueron consolidados con la creación del Espacio Europeo de Educación Superior (EEES) en 1999, que además 
trajo la consiguiente revisión de los enfoques educativos, lo que dio lugar a un importante cambio en la estrategia docente y situó al estudiante como protagonista de la enseñanza y el aprendizaje "a lo largo de la vida" (Hernández-Pina, 2014).

Inicialmente, la fuerte e imperante idea sobre la autonomía universitaria planteó dudas y escepticismo sobre la implantación de los procedimientos de evaluación, aunque poco a poco esta tensión se fue diluyendo y se posibilitó la impregnación de la cultura de la calidad en todos los niveles (Fernández Lamarra, 2012). La evaluación externa comenzó siendo testimonial mediante la incorporación de evaluadores que eran externos al sistema universitario y que llegaron a asumir el papel de ser cómplices de los autoinformes realizados por las instituciones, ya que no tenían suficientes directrices para aplicar la evaluación de los estándares de referencia (Muñoz-Cantero y Pozo, 2014). La Fundación Agencia Nacional de Evaluación de la Calidad y Acreditación (ANECA) fue creada el 19 de julio de 2002 por acuerdo de Consejo de Ministros con el objetivo de promocionar y asegurar la Calidad del Sistema de Educación Superior en España para contribuir al desarrollo del EEES. Dicha promoción y aseguramiento de la calidad se realizaría mediante la aplicación de procesos de evaluación, certificación y acreditación (Real Decreto 1112/2015, de 11 de diciembre).

Con el desarrollo de la LOU 6/2001, la LOMLOU 4/2007 y el Real Decreto $1393 / 2007$ se establecieron las bases para las nuevas titulaciones y se definieron los términos para llevar a cabo las acreditaciones de las mismas conforme a "la verificación del cumplimiento del proyecto presentado por la Universidad" (Real Decreto 1393/2007, Preámbulo). Todo ello llevó a implantar los Sistemas de Garantía Interna de Calidad (SGIC) en las instituciones universitarias, actualmente denominados como SAIC (Sistemas de Aseguramiento Interno de Calidad), y a la creación de unidades con personal técnico para la realización de los procedimientos entorno a la Calidad. Estos sistemas están dentro del proceso de transparencia exigida por el EEES para garantizar que las actuaciones emprendidas en torno a la Educación Superior van en la dirección apropiada a la consecución de los objetivos establecidos (Ríos de Deus, 2014). Los SAIC están constituidos en base al cumplimiento de la normativa estatal y conforme a los Criterios y directrices para el Aseguramiento de la Calidad en el Espacio Europeo de Educación Superior (ENQA, 2015) creados en la reunión de Ministros de Educación de la Unión Europea de Bergen en 2005 y modificados y aprobados por la conferencia celebrada en Ereván en 2015.

Actualmente, la práctica totalidad de las universidades y, por lo 
tanto, sus titulaciones, está implicada en procesos de evaluación y acreditación. Por lo tanto, el objetivo de crear el EEES se ha alcanzado (Michavila Pitarch, 2011) y se avanza lentamente en la implantación de una cultura de calidad y de evaluación en el sistema universitario español y en la puesta en marcha de instrumentos y sistemas para poder llevarla a cabo (Sánchez Pérez, 2007). A través del programa ACREDITA de la ANECA se realiza la evaluación de la renovación de la acreditación de las titulaciones conforme a la legislación española y según los ciclos establecidos para cada titulación $(6,7$ y 8 años para grados de 240, 300 y 360 créditos respectivamente, 4 años para másteres y 6 años para doctorados) con dos objetivos claros (ANECA, 2020):

1) Constatar que la impartición de la titulación se está llevando a cabo en los términos que se establecieron en su proyecto inicial, y gracias a los cuales la titulación obtuvo la condición de oficial.

2) Confirmar que la titulación contribuye a la formación de los estudiantes y a la consecución de los objetivos definidos.

En la aplicación de estas evaluaciones se han detectado, sin embargo, diferentes dificultades y obstáculos que están relacionados con varios aspectos (Vázquez García, 2015). En primer lugar, existe poca cultura de la evaluación, que genera un clima de desconfianza hacia la eficacia de estos procesos y da lugar a que sean vistos con recelo por una parte de los grupos de interés que intervienen en ellos. Esto, sumado a la falta del reconocimiento del empeño de la labor académica que conlleva la realización de los procesos, hace que las personas que participan desarrollen la evaluación como una opción de compromiso hacia la institución. En segundo lugar, no se ha generado un sistema de información y formación que dé acceso a los datos esenciales de los procesos de evaluación y acreditación. La comunidad universitaria desconoce los diferentes procesos de evaluación y las metodologias empleadas. Estos motivos han provocado que exista una escasa participación de los diferentes grupos de interés en los procesos de evaluación y acreditación (De Miguel y Apodaca, 2009). Sin duda, estas dificultades tienen su origen en la falta de financiación necesaria para generar una estructura de personal y recursos enfocados a la planificación, realización, puesta en común e información sobre los procedimientos de evaluación realizados y a la evaluación de fortalezas y debilidades, y ejecución de modificaciones y propuestas de mejora.

Según el informe de cifras de evaluación de ANECA para la verificación, seguimiento y la renovación de la acreditación de las titulaciones universitarias, en el periodo 2008-2016 se realizaron 2.413 evaluaciones de verificación en títulos de Grado, 3.742 en 
títulos de Máster y 624 en Programas de Doctorado, de los cuales 171 Grados y 281 Másteres obtuvieron la evaluación de renovación de la acreditación favorable del 2013 al 2016. Por lo tanto, desde el 2017 hasta la actualidad, se están evaluando y acreditando a un gran número de titulaciones.

Todas las universidades españolas han de someter sus titulaciones a procesos de evaluación y acreditación, si bien cabe plantearse cómo afronta el profesorado estos procedimientos y si tiene en su mano las herramientas necesarias y toda la información y formación requeridas para su aplicación. Hay escasa documentación sobre la opinión de los actores implicados en los diferentes aspectos de estos procesos, especialmente del profesorado, y se hace necesario conocer su percepción con el fin de saber si la aplicación de estos procedimientos de evaluación y acreditación realmente incide en la mejora de la calidad de las propias titulaciones y de la institución.

\section{Método}

\section{Objetivos}

Los objetivos de este trabajo fueron:

1. Analizar la percepción del profesorado sobre los procesos de evaluación y acreditación de las titulaciones, en cuanto a las facilidades y dificultades detectadas en su aplicación.

2. Comprobar el grado de liderazgo ejercido por los equipos de dirección de los centros y los coordinadores de titulaciones en dichos procesos.

\section{Diseño}

Se implementó un enfoque descriptivo de corte mixto pues se emplearon técnicas e instrumentos de recogida de información de carácter cuantitativo y cualitativo. En concreto, se empleó un diseño explicativo secuencial, DEXPLIS (Creswell, 2012), en el que inicialmente se recabaron y analizaron los datos cuantitativos, y los resultados obtenidos se refrendaron mediante los obtenidos con los datos cualitativos.

\section{Participantes y contexto}

Esta investigación contó con dos muestras pues se recogió información con técnicas cuantitativas y cualitativas. Por un lado, participaron 203 profesores de la Facultad de Educación de una 
universidad estatal que impartieron clase durante el curso académico 2017-2018. Esta fue una muestra representativa de la población objeto conformada por 334 profesores con un error del $5 \%(a=.05, z=1.96, p$ $=q=.5)$. Del total de profesorado participante, 83 eran profesores asociados (40.88\%), 8 Ayudantes Doctor (3.94\%), 51 Contratados Doctor $(25.12 \%), 52$ Profesores Titulares $(25.62 \%)$ y 9 Catedráticos (4.43\%). Además, 104 individuos (51.23\%) habian ocupado un cargo de gestión en alguna ocasión y/o habian participado en la Comisión de Calidad o de alguna titulación del centro, frente a $99(48.77 \%)$ que no lo había hecho en ninguna ocasión.

Para la recogida de datos cualitativos se hizo una selección deliberada e intencional con el fin de tener la máxima representatividad por categoría profesional y en cuanto al hecho de haber ocupado un cargo de gestión o haber participado en la Comisión de Calidad o de las titulaciones del centro. Cinco profesores accedieron a ser entrevistados voluntariamente, cuyos perfiles fueron codificados de la siguiente manera:

- Código TUED: profesora Titular de Universidad, mujer con 30 años de experiencia docente, que había ocupado cargos de gestión y había participado en la Comisión de Calidad de la Facultad de Educación.

- Código TUCA: profesor Titular de Universidad, varón con 26 años de experiencia docente, que ocupó un cargo de gestión en el área de Calidad.

- Código CDCG: profesor Contratado Doctor, varón con 8 años de experiencia docente, que había ocupado un cargo en una titulación de la Facultad de Educación.

- Código TUCO: profesor Titular de Universidad, varón con 37 años de experiencia docente, que había sido miembro de la Comisión de Calidad del Centro.

- Código ASOC: profesor Asociado, varón con 6 años de experiencia docente, que nunca había ocupado cargos de gestión, ni había formado parte de la Comisión de Calidad o de las titulaciones.

Con la implantación de la nueva normativa y la creación del EEES, la Facultad de Educación de este estudio dio el paso de las antiguas diplomaturas y licenciaturas a los actuales grados y másteres. Los nuevos planes de estudios fueron verificados por ANECA en 2009 (en el caso de los grados en Educación Infantil, en Educación Primaria, en Educación Social, y en Pedagogia), y en 2010, 2013 y 2014 (en el caso de sus 8 másteres oficiales). Tras varias evaluaciones de monitorización de las titulaciones, y tramitadas las solicitudes y aprobaciones de modificaciones de las memorias de algunas titulaciones, el centro superó con éxito la evaluación de la renovación de la acreditación de toda su oferta de estudios de 2015 a 2018. Esta acreditación es una 
garantía de cumplimiento de criterios y estándares, a la vez que asegura la adquisición de competencias por parte del estudiante para una mejor incorporación al mercado laboral y para satisfacer las necesidades de la sociedad en su conjunto (Fueyo Gutiérrez, 2004).

\section{Instrumentos}

En esta investigación se recogieron datos mediante una escala Likert y entrevista individual. Por un lado, se administró una escala Likert ad hoc de cinco valores (totalmente de acuerdo, de acuerdo, indiferente, en desacuerdo, totalmente en desacuerdo). Inicialmente, los items de la escala fueron sometidos a juicio de expertos, lo que llevó a una reducción del número inicial de 41 a 35 ítems. Se calculó la fiabilidad y consistencia interna mediante el Alfa de Cronbach y se obtuvo un resultado de $a=.85$. Según George y Mallery (2003) este resultado es "bueno", considerándose excelente a partir de $a=.9$. Seguidamente, se realizó una validez de constructo mediante un análisis factorial de componentes principales con rotación varimax. Se obtuvo un $p$-valor significativo de .000 para la prueba de esfericidad de Bartlett y un coeficiente de Kaiser-Meyer-Olkin (KMO) para la proporción de la varianza que tienen en común las variables analizadas de .853, coeficiente cercano a la unidad (se considera una buena adecuación muestral a partir de .5). Considerando únicamente los eigenvalues por encima de 1, el análisis factorial mostró una clasificación de los ítems en 9 dimensiones, explicando un $63.51 \%$ de la varianza total.

Además, se diseñó un guion de entrevista ad hoc para llevar a cabo entrevistas en profundidad semiestructuradas a nivel individual tras la obtención del consentimiento informado de los participantes. Se formularon preguntas sobre el conocimiento de los procesos de evaluación y acreditación, la participación en ellos, el procedimiento seguido en estos, etc. Con preguntas abiertas de este tipo se consiguió recabar datos de corte cualitativo que permitieron obtener información individualizada y personal sobre sus experiencias, opiniones, valoración $\mathrm{y}$ actitud frente a las situaciones (Massot, Dorio, y Sabariego, 2014).

\section{Procedimiento}

Se concertó una reunión con el Decano y el Secretario de la facultad para presentar el objetivo de la investigación y solicitar permiso para realizar una encuesta general a todo el profesorado de esa facultad sobre los procesos de evaluación y acreditación. Se tuvo en cuenta los criterios éticos en la elaboración del instrumento de recogida de datos y 
en su aplicación al profesorado. Seguidamente, se procedió al envío de la escala a través de la plataforma habilitada por la universidad para estos efectos.

Con respecto a las entrevistas, se contactó con los cinco informantes vía correo electrónico, y tras su aceptación a participar, se concertaron las citas para su realización. Tras el proceso de transcripción, se les remitió esta para que tuvieran opción de confirmar o modificar algún aspecto. Realizada la revisión correspondiente, y recibido su consentimiento, se procedió a su análisis mediante la generación de códigos a partir de las temáticas más repetidas durante las entrevistas, así como al registro de frecuencias y porcentajes de ocurrencia de los códigos.

\section{Análisis de la información}

Los datos cuantitativos recogidos mediante la escala Likert se procesaron y analizaron con el paquete estadístico de software libre $R$. Para la búsqueda de diferencias significativas en los ítems según variables sociodemográficas, se aplicaron tests no paramétricos, por ser estos test los más robustos para datos ordinales. En concreto, se empleó la $U$ de Mann-Whitney para variables independientes con dos niveles de respuesta y el test de Kruskal-Wallis (ANOVA no paramétrica) para variables con más de dos niveles (se tomó p-valor inferior a .05 y nivel de significación $a=.05$ ). Para el post-hoc, se realizó el Pairwise Wilcoxon Rank Sum Test con corrección de Bonferroni. Los datos se analizaron en función de la categoría profesional del profesorado y del hecho de haber ocupado un cargo de gestión.

En cuanto a los datos textuales recogidos mediante la entrevista se analizó el contenido de las cinco entrevistas con el programa Atlas.ti v.8.1.28.0 para Windows (Scientific Software Development GmbH, 2017) y se identificaron códigos.

\section{Resultados y discusión}

\section{Percepción sobre los procesos de evaluación y acreditación}

El análisis de la percepción del profesorado sobre los procesos de evaluación y acreditación puso de manifiesto las dificultades y facilidades a las que se enfrenta el profesorado a la hora de participar en dichos procesos (Figura 1). Se observó un alto grado de acuerdo entre los encuestados sobre el hecho de que estos procesos deben implicar a todo el profesorado de la facultad (67.5\% en item 5), y que es muy complicado recopilar todas las evidencias que se solicitan en el 
proceso (66.5\% en ítem 2). Además, un $61.6 \%$ opinó que es muy dificil contar con alumnado egresado y los empleadores (item 4), y que aunque es un proceso complejo y laborioso, merece la pena porque ayuda a mejorar las titulaciones (item 8). Por el contrario, el 39.4\% de los participantes consideró que no tienen la suficiente formación e información para colaborar en estos procesos (ítem 10) y el $27.1 \%$ de los participantes estuvo en desacuerdo o totalmente desacuerdo con la idoneidad de los evaluadores externos que intervienen en la evaluación (item 3). Asimismo, un $60.1 \%$ de los encuestados indicó que se debería aumentar el personal dedicado exclusivamente al apoyo en la implementación de estos procesos (ítem 7).

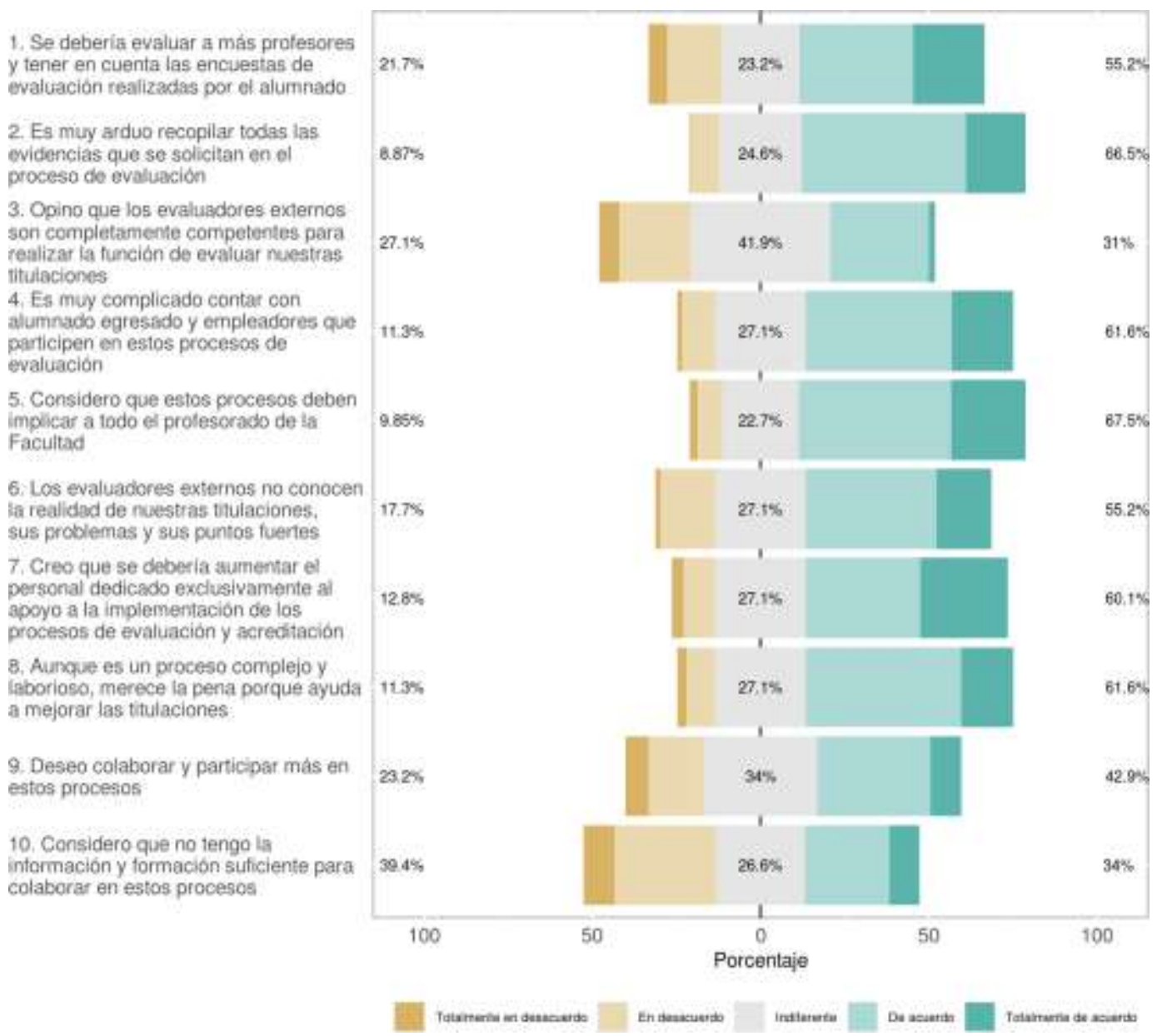

Figura 1. Percepción del profesorado sobre facilidades y dificultades identificadas en los procesos de evaluación y acreditación 
En cuanto a la existencia de diferencias estadísticamente significativas en los ítems que median la percepción del profesorado entre los docentes participantes en función de la categoría profesional y de haber ocupado un cargo de gestión, las mayores diferencias se observaron entre profesorado asociado y el resto de categorías profesionales (Tabla 1).

Tabla 1

Diferencias de percepción entre profesorado en función de la categoría profesional y de haber ocupado un cargo de gestión

\begin{tabular}{|c|c|c|c|}
\hline Cuestión & $\begin{array}{l}p \text {-valor } \\
\text { según } \\
\text { categoría }\end{array}$ & $\begin{array}{l}\text { Diferencias según } \\
\text { profesional }\end{array}$ & $\begin{array}{l}p \text {-valor } \\
\text { según } \\
\text { cargo }\end{array}$ \\
\hline Îtem 1 & $.007^{*}$ & $\begin{array}{l}\text { Asociado } \quad(\bar{x}=3.843) \quad \text { con } \\
\text { Contratado Doctor }(\bar{x}=3.196)\end{array}$ & .960 \\
\hline Ítem 2 & $.019^{*}$ & $\begin{array}{l}\text { Asociado } \quad(\bar{x}=3.554) \quad \text { con } \\
\text { Contratado Doctor }(\bar{x}=3.961)\end{array}$ & .086 \\
\hline Ítem 3 & .600 & - & .550 \\
\hline Ítem 4 & $.002^{*}$ & $\begin{array}{l}\text { Asociado } \quad(\bar{x}=3.386) \quad \text { con } \\
\text { Contratado Doctor }(\bar{x}=3.863) \text { y } \\
\text { con Profesor Titular }(\bar{x}=3.942)\end{array}$ & $\begin{array}{l}.001^{*} \quad(\text { No } \\
\bar{x}=3.513, \text { Sí } \\
\bar{x}=3.960)\end{array}$ \\
\hline Ítem 5 & .340 & - & .470 \\
\hline Ítem 6 & $.008^{*}$ & $\begin{array}{lll}\text { Asociado } & (\bar{x}=3.578) \quad \text { con } \\
\text { Catedrático }(\bar{x}=2.556) \text { y } \\
\text { Contratado Doctor }(\bar{x}=3.804) \\
\text { con Catedrático }(\bar{x}=2.556)\end{array}$ & .190 \\
\hline Ítem 7 & $.033^{*}$ & $\begin{array}{l}\text { Asociado } \quad(\bar{x}=3.554) \quad \text { con } \\
\text { Contratado Doctor }(\bar{x}=4.039)\end{array}$ & $\begin{array}{l}.0420^{*} \quad(\text { No } \\
\bar{x}=3.598, \text { Sí } \\
\bar{x}=3.824)\end{array}$ \\
\hline Ítem 8 & .100 & - & .640 \\
\hline Ítem 9 & $.043^{*}$ & $\begin{array}{l}\text { Asociado } \quad(\bar{x}=3.470) \quad \text { con } \\
\text { Contratado Doctor }(\bar{x}=3.000)\end{array}$ & .680 \\
\hline Ítem 10 & $.000^{*}$ & $\begin{array}{l}\text { Asociado }(\bar{x}=3.434) \\
\text { Contratado Doctor }(\bar{x}=2.804), \\
\text { con Profesor Titular }(\bar{x}=2.385) \\
\text { y con Catedrático }(\bar{x}=1.889)\end{array}$ & $\begin{array}{l}.000^{*} \quad(\mathrm{No} \\
\bar{x}=3.359, \mathrm{Si} \\
\bar{x}=2.365)\end{array}$ \\
\hline
\end{tabular}

Nota. Véase el enunciado de los items en la Figura 1.

Los valores con asterisco indican un nivel de significación inferior a .050.

De las entrevistas realizadas para ahondar en la identificación de facilidades y dificultades, se generaron códigos y se calculó la frecuencia y porcentaje de ocurrencia. Destacan los códigos de Pocos 
medios humanos (25\%, $f=15)$, Exigencias ANECA (16.7\%, $f=10)$, Poca formación $(15 \%, f=9)$ y Pocos recursos materiales $(13.3 \%, f=8)$, así como la referencia a los escasos recursos humanos y materiales con los que se cuenta para llevar a cabo la tramitación de los procesos de evaluación y acreditación y la poca formación del personal:

"El trabajo recae muchísimo en los centros, y los centros no tienen ni los recursos humanos ni materiales para hacer frente a la ingente cantidad de documentación que se está haciendo" (TUED).

"Hay una dificultad que es la de la capacitación o la formación del profesorado en este tipo de procesos. No todo el profesorado está preparado, o requeriría de un cierto aprendizaje para abordar estos procesos" (TUCA).

"Habría que tener más medios humanos, no digo ya presupuestarios sino más personal tanto en cargos de gestión como personal de administración y servicios, y más figuras docentes que tuviéramos una dedicación no digo exclusiva, pero que pudiéramos atender mejor esto, con más tiempo" (CDCG).

Cuando los encuestados fueron preguntados sobre posibles facilidades en la participación en los procesos de evaluación y acreditación, sobresale el apoyo de la Unidad para la Calidad de la universidad por su asesoramiento y ayuda en la recopilación de todas las evidencias para la generación de los documentos solicitados por ANECA, como ilustran las siguientes citas:

"Las facilidades han partido de personas concretas de la Unidad para la Calidad. Y muchas veces estas personas también han estado sometidas a los vaivenes de la falta de formación y de información que ha habido en el proceso" (TUED).

"La facilidad es que está todo establecido, los mecanismos, te dicen lo que tienes que hacer, y tú haces lo que te dicen. Entonces, si hay que hacer una memoria, ves los apartados que debe tener y los haces. Es decir, tú no te planteas realmente el problema" (TUCO).

Este estudio identificó tres grandes dificultades a la hora de la realización de los procesos de evaluación y acreditación: complejidad del modelo, escasez de recursos y ausencia de participación fluida. En primer lugar, la complejidad del modelo seguido para la evaluación y acreditación de las titulaciones. El modelo actual requiere conocimiento y dominio, es decir, un aprendizaje intencionado. Se podría pensar que al tratarse de profesorado universitario, todo lo que tenga que ver con la evaluación ya está dominado, al menos de manera intuitiva. Sin 
embargo, la estructuración de los procesos de evaluación y acreditación es exigente y abarca múltiples y diversas variables que afectan a las titulaciones (De Miguel y Apodaca, 2009). Habría que partir del principio de que el profesorado no tiene por qué conocer la naturaleza de cómo se crean, cómo se procesan todos los datos que hay que recoger y analizar. Efectivamente, la recogida y generación de los datos son tareas de unidades y de personal técnico asignados a los servicios de Calidad de las universidades. Se trata de un grupo de técnicos reducido que facilita y asesora a todas las facultades de la universidad. Asimismo, el propio centro ha de generar parte de esos datos, y esta es una tarea técnicamente especializada que requiere un cierto conocimiento. Por lo tanto, la capacitación o formación del profesorado en este tipo de procesos es en sí una dificultad (Fernández Lamarra y Aiello, 2014).

Por otro lado, existe escasez de recursos. Los procesos de evaluación de la calidad externa e interna son muy costosos en cuanto a tiempo y esfuerzo. Asimismo, los recursos humanos son muy limitados en cuanto al personal técnico que procesa la información y al profesorado que participa de una manera u otra en distintos niveles (departamento, comisiones de calidad, centro). Los resultados revelaron que se debería aumentar el volumen del personal técnico y repensar el perfil de las personas que participan en los procesos de evaluación y acreditación (Rodríguez Espinar, 2013). El alto porcentaje de profesorado asociado está contratado a tiempo parcial y eso significa que ese profesorado no puede ni tiene por qué dedicarse a tiempo completo a estas labores. Su implicación en tareas de investigación y de gestión de los procesos de calidad se ve, no solo dificultada, sino anulada. "No hay reforma sin recursos y la de Bolonia los requería indudablemente" (Vázquez García, 2011, p. 36).

Por último, la ausencia de participación fluida se detectó como otra dificultad. Existen comisiones de calidad del centro, de titulaciones, consejos de departamentos, Junta de Facultad, etc., que están interconectadas e interrelacionadas. Sin embargo, la realidad en el día a día en los procesos de calidad es que la implicación del personal que forma parte de las comisiones y que, por tanto, asume más específicamente la responsabilidad de realizar o participar en las tareas de calidad, está limitada en términos de compromiso y continuidad en la dedicación (Sánchez Pérez, 2007). Los inexistentes estímulos proporcionados al profesorado para el desarrollo de unas tareas que requieren mayores dosis de esfuerzo y que demandan mayores niveles de implicación, junto con unos precarios recursos, comprometen el éxito de estos procesos de evaluación (Vázquez García, 2011), así que no es de extrañar el resultado hallado. 


\section{Percepción sobre el papel desempeñado por los cargos de gestión y las coordinaciones de titulación en los procesos de evaluación y acreditación}

El análisis descriptivo de los ítems que medían la percepción de los participantes sobre el papel de los cargos de gestión y miembros de comisiones de calidad (Figura 2) reveló que el $62.6 \%$ de los participantes opinaron que el Equipo Decanal y los coordinadores de las titulaciones son los que lideran y controlan los procesos de evaluación y acreditación (ítem 14). Además, un 52.2\% opinó que los cargos de gestión y las coordinaciones de titulación están implicados en la realización de la evaluación pues motivan al resto del profesorado (item 11), si bien hay división de opiniones sobre la información que estos cargos proporcionan al profesorado (item 12). Finalmente, un $52.2 \%$ de los encuestados remarcó que habría que implicar a más profesorado en los procesos de evaluación (ítem 13).

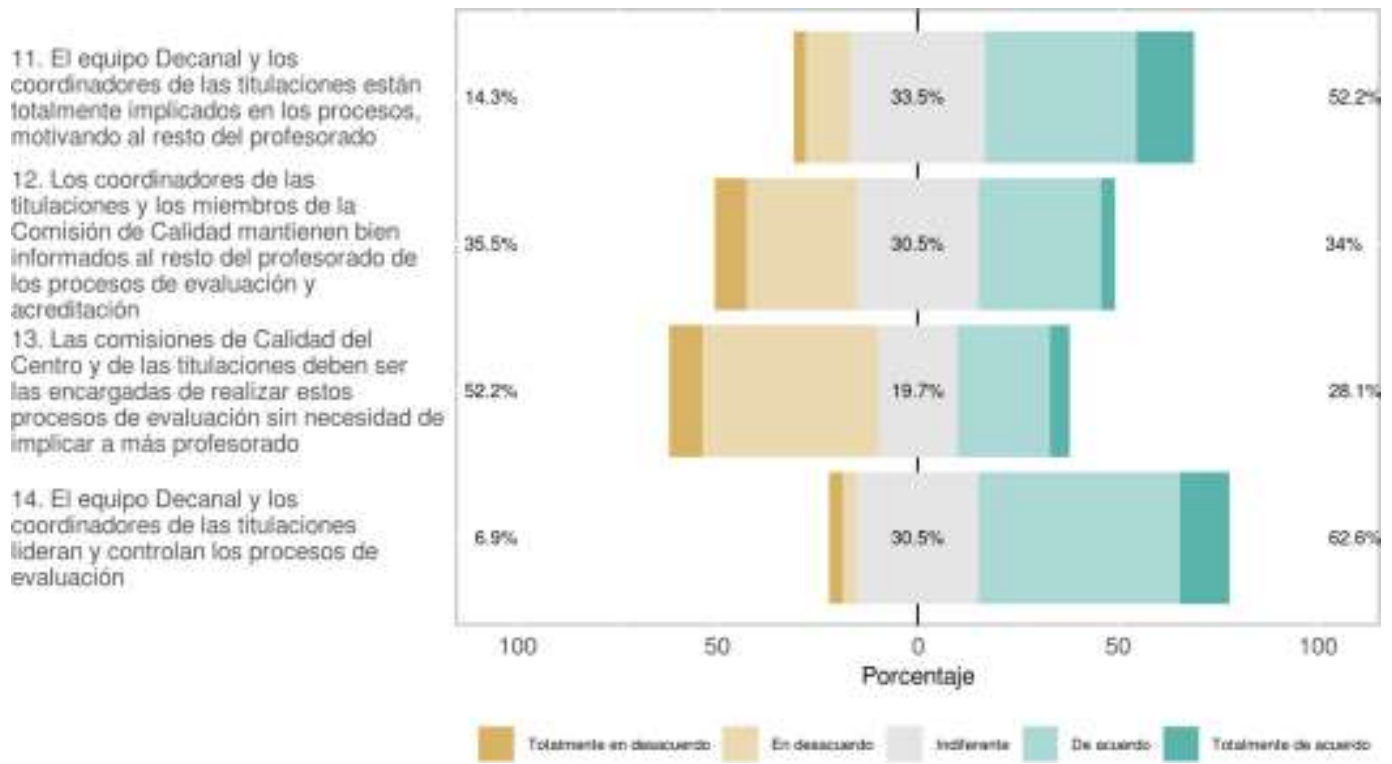

Figura 2. İtems que miden la percepción del profesorado sobre la actuación de los cargos de gestión y los coordinadores de titulaciones

En cuanto a la existencia de diferencias estadísticamente significativas en estos items según categoria profesional y haber ocupado un cargo de gestión solo se detectaron diferencias según esta última variable en el ítem 12, teniendo mayor media aquellos 
participantes que nunca habian ocupado un cargo de gestión $(\bar{x}=2.94)$ frente a aquellos que sí lo han hecho $(\bar{x}=2.59)$.

De las entrevistas realizadas para ahondar en este sentido, se generaron códigos junto con sus frecuencias y porcentajes entre los que destacaron los códigos de Trabajo en equipo $(28.1 \%, f=9)$, Cargo de gestión $(25 \%, f=8)$, y Miembro de comisión $(21.9 \%, f=7)$. Además, los participantes resaltaron el compromiso de los cargos y los miembros de comisiones de calidad con la correcta realización de estos procesos:

"Conocí los procesos a raíz de mi cargo como coordinador de titulación. No es un conocimiento tampoco en profundidad, sino que viene siempre dictado por el Vicedecanato de Ordenación y Calidad, y la propia Unidad de Calidad" (CDCG).

"He participado en otros procesos como miembro de una comisión, como uno más, implicándome para conseguir una evaluación positiva" (TUCO).

"Son los miembros de las comisiones de Calidad de las titulaciones los que se ocupan de la realización de estos procesos de evaluación y acreditación" (ASOC).

Tal y como era de esperar, se percibe que el equipo Decanal del centro y los coordinadores de las diferentes titulaciones son los máximos responsables del control de los procesos de evaluación y acreditación de las titulaciones, y lideran estos procesos, aunque existe un sentimiento de desinformación. Estos cargos tienen un mejor conocimiento de todo el procedimiento y están implicados para que las titulaciones superen positivamente todas las evaluaciones a las que son sometidas. Además, son los responsables de aplicar las propuestas de mejora o las posibles modificaciones que resulten de la evaluación final, buscando siempre la mejora de la calidad de las titulaciones. Bajo su responsabilidad, el reto actualmente está en llegar a ser líder de líderes mediante la promoción de una estructura que facilite que los miembros del centro puedan desarrollar de una manera creativa sus competencias y permitiendo que todos trabajen en un proyecto común. El liderazgo distribuido en el colectivo docente reconoce que diferentes personas, y no necesariamente aquellas que ocupan puestos de gestión en la organización de los centros, pueden emerger como fuentes de influencia (Bolivar, 2011). Desde este punto vista, deben intentar implicar, no solo al profesorado, sino también al resto de grupos de interés como son alumnado, egresados, empleadores y personal de administración y servicios en la participación en los procesos de evaluación y acreditación de las titulaciones. 


\section{Conclusiones}

Esta investigación tuvo por objetivos analizar la percepción del profesorado sobre los procesos de evaluación y acreditación de las titulaciones ofertadas por la Facultad de Educación de una universidad pública española, y comprobar el grado de liderazgo ejercido por los equipos de dirección de los centros y los coordinadores de titulaciones en dichos procesos. Los datos obtenidos invitan a reflexionar sobre las dificultades detectadas en la aplicación de los modelos de evaluación y acreditación en la universidad española. Aunque estos procesos son fundamentales en la mejora de la calidad de las titulaciones y las instituciones universitarias, y a pesar de que hay grandes avances en la interiorización de la cultura de la evaluación, es necesario hacer una revisión de los procedimientos implementados que simplifique y mejore su aplicación. Esto permitiria que los procesos fueran más cercanos a los diferentes grupos de interés. Así, de este estudio se concluye, en consonancia con otras investigaciones, que estos procesos son percibidos como procedimientos burocratizados que implican a personal con escasa formación en los mismos (Buendía Espinosa, 2011; Monarca, Thoilliez, Garrido, y Prieto, 2018; Zapata y Tejeda, 2009). Se necesitan, por tanto, personas formadas en las unidades de gestión de la Calidad, que cumplan la función de ejecución de estos procedimientos y de las numerosas acciones que es necesario desarrollar, aligerando la carga que soporta el profesorado dedicado a estas tareas, que a menudo está sobrepasado ante los compromisos académicos de gestión y con la institución, y que desemboca en la desatención de las tareas docentes e investigadoras propias de su figura académica (De Miguel y Apodaca, 2009). Todo ello requiere de la correspondiente financiación para hacer frente a la falta de recursos humanos y materiales que evidentemente son necesarios para el correcto funcionamiento en la aplicación de los procesos.

Tal y como revela este estudio la escasa participación activa, el limitado deseo de colaborar y la poca intención de formarse para ser un experto en Calidad son algunas de las grandes dificultades a la hora de llevar a cabo los procesos de evaluación y acreditación. La participación sostenida, activa y comprometida en las tareas de análisis al más alto nivel del centro es reducida. La persona que coordina, gestiona, dirige todas estas tareas, las cuales implican recoger y sistematizar datos, elaborar informes, comunicar resultados, ordenarlos, analizarlos, interpretarlos y tomar decisiones, es la que suele tener que asumir ese trabajo casi aisladamente. Son estas tareas técnicamente muy complejas que requieren un fuerte compromiso. La mayoría del profesorado huye de implicarse en dichas tareas, aun cuando es 
miembro de una comisión de Calidad, y reconoce que son los responsables del equipo directivo del centro y de las titulaciones los que soportan esta carga de trabajo.

Finalmente, esta investigación pone de manifiesto la necesaria realización de acciones de formación e información sobre estos procesos con el fin de que el profesorado comprenda la importancia y los beneficios de la evaluación y acreditación de las titulaciones para su facultad. Esto permitiría conseguir una mayor implicación y coordinación de todos los implicados en los mismos, y fomentaría la participación de un mayor número de docentes, que redundaría en el reparto de tareas y responsabilidades, y en una mejor ejecución de las mismas.

Con respecto a las limitaciones detectadas de este estudio, se contó con una muestra limitada tanto en la recogida de datos mediante la escala como en la participación en las entrevistas, donde solo se consiguió la realización de cinco entrevistas. Es por ello que de ninguna manera debe generalizarse los resultados obtenidos en este estudio al resto de profesorado del centro u otras facultades.

Se debe remarcar la importancia que los procesos de evaluación y acreditación de las titulaciones universitarias tienen en el avance hacia una gestión transparente y como deber de rendición de cuentas a la sociedad, a pesar de los defectos y complejidades que entrañan. Por ello, es primordial implicar a todos los grupos de interés para conseguir una mejora real y genuina, y que la calidad total llegue a todos los ámbitos.

\section{Referencias}

ANECA (2020). ANECA. Recuperado de http://www.aneca.es/ANECA

Bolivar, A. (2011). Aprender a liderar líderes. Competencias para un liderazgo directivo que promueva el liderazgo docente. Educar 2011, 47(2), 253-275.

Comunicado de Bergen (2005). El Espacio Europeo de Educación Superior. Alcanzando las metas. Recuperado de http://media.ehea.info/file/2005_Bergen/53/8/2005_Bergen_ Communique_Spanish_580538.pdf

Comunicado de Everán (2015). Recuperado de http://media.ehea.info/file/2015_Yerevan/70/7/YerevanCom muniqueFinal_613707.pdf

Creswell, J. (2012). Educational research: Planning conducting and evaluating quantitative and qualitative research (4. ${ }^{\mathrm{a}}$ ed.). 
Massachusett: Pearson.

De Miguel, M., \& Apodaca, P. (2009). Criterios para evaluar el impacto de los Planes de Evaluación de la Calidad de la Educación Superior. Revista de Educación, 349, 295-310. Disponible en https: / /dialnet.unirioja.es/servlet/articulo?codigo $=2995075$

ENQA (2015). Standards and Guidelines for Quality Assurance in the European Higher Education Area. Brussels, Belgium. Recuperado de https://enqa.eu/index.php/home/esg/

Fernández Lamarra, N. (2012). La Educación Superior en América Latina. Aportes para la construcción de una nueva agenda. Revista Debate Universitario, 1(1), 1-29. Disponible en https: / /dialnet.unirioja.es/servlet/articulo?codigo $=4859695$

Fernández Lamarra, N., \& Aiello, M. (2014). La evaluación universitaria en Argentina en el marco de América Latina. Situación y desafíos en relación al aseguramiento de la calidad. En H. Monarca y J. Valle (Coords.), Evaluación de la calidad de la Educación Superior en Iberoamérica (pp. 21-48). Madrid: GIPES-UAM.

Fueyo Gutiérrez, A. (2004). Evaluación de titulaciones, centros y profesorado en el proceso de Convergencia Europea: ¿de qué calidad y de qué evaluación hablamos? Revista Interuniversitaria de Formación del Profesorado, 18(3), 207-2019. Recuperado de https: / /dialnet.unirioja.es/servlet/articulo?codigo $=1113392$

George, D., \& Mallery, P. (2003). SPSS for Windows step by step: A Simple Guide and Reference. 11.0 Update (4. ${ }^{\mathrm{a}}$ ed.). Boston: Allyn \& Bacon.

Hernández-Pina, F. (2014). Evaluación y acreditación del profesorado, programas e instituciones educativas. Revista Electrónica Interuniversitaria de Formación del Profesorado, 17(1), 15-32. http://dx.doi.org/10.6018/reifop.17.1.198821

Ley Orgánica 6/2001, de 21 de diciembre, de Universidades. Boletín Oficial del Estado, 307, de 24 de diciembre de 2001, pp. 49400-49425. Recuperado de https: / / boe.es/boe/dias/2001/12/24/pdfs/A49400-49425.pdf

Ley Orgánica 4/2007, de 12 de abril, por la que se modifica la Ley Orgánica 6/2001, de 21 de diciembre, de Universidades. Boletín Oficial del Estado, 89, de 13 de abril de 2007, pp. 16241-16260. Recuperado de

https: / / boe.es/boe/dias/2007/04/13/pdfs/A16241-16260.pdf 
Massot, I., Dorio, I., \& Sabariego, M. (2014). Estrategias de recogida y análisis de la información. En R. Bisquerra et al., Metodología de la investigación educativa (4. ${ }^{\text {a }}$ ed.) (pp. 329-365). Madrid: La Muralla.

Michavila Pitarch, F. (2011). Bolonia en crisis. Revista de Docencia Universitaria. REDU, 9(3), 15-27. Recuperado de http: / / redaberta.usc.es/redu

Muñoz-Cantero, J. M., \& Pozo, C. (2014). El escenario de la calidad en la Universidad española: de dónde venimos y hacia dónde vamos. Revista Electrónica Interuniversitaria de Formación del Profesorado, 17(3), 1-16. http://dx.doi.org/10.6018/reifop.17.3.204011

$\mathrm{R}$ Core Team (2020). R: A Language and Environment for Statistical Computing. Viena: R Foundation for Statistical Computing.

Real Decreto 1112/2015, de 11 de diciembre, por el que se aprueba el Estatuto del Organismo Autónomo Agencia Nacional de Evaluación de la Calidad y Acreditación. Boletín Oficial del Estado, 18 de diciembre de 2015, núm. 302.

Real Decreto 1393/2007, de 29 de octubre, que establece la ordenación de las enseñanzas universitarias oficiales. Boletín Oficial del Estado, 30 de octubre de 2007, núm. 260.

Ríos de Deus, M.P. (2014). Sistemas de garantía de calidad en centros de Educación Superior. Revista Electrónica Interuniversitaria de Formación del Profesorado, 17(3), 17-34. http: //dx.doi.org/10.6018/reifop.17.3.204021

Rodriguez Espinar, S. (2013). Panorama Internacional de la Evaluación de la Calidad en la Educación Superior. Madrid: Sintesis.

Sánchez Pérez, J. I. (2007). Evaluación de centros y calidad. Revista de la Asociación de Inspectores de Educación en España, 5, 1-5. Disponible en https://www.researchgate.net/publication/28144555_Evaluac ion_de_centros_y_calidad

Scientific Software Development GmbH (2017). Atlas.ti for Windows, Versión 8.1.28.0. Berlín: Scientific Software Development GmgH.

Vázquez García, J. A. (2015). Nuevos escenarios y tendencias universitarias. Revista de Investigación Educativa, 33(1), 13-26. http://doi.org/10.6018/rie.33.1.211501

Zapata, G. \& Tejeda, I. (2009). Impactos del aseguramiento de la calidad y acreditación de la educación superior. Calidad en la educación, 31, 192-209. Recuperado de https://dialnet.unirioja.es/servlet/articulo?codigo $=3214471$ 